\title{
Atomic Scale Structure and Defects in 2D GaSe Films and Van der Waals Interface
}

\author{
Jared M Johnson $^{1}$, Choong Hee Lee ${ }^{2}$, Sriram Krishnamoorthy ${ }^{2}$, Siddharth Rajan ${ }^{2}$ and Jinwoo Hwang ${ }^{1}$ \\ ${ }^{1 .}$ Department of Materials Science and Engineering, The Ohio State University, Columbus, OH 43212 \\ 2. Department of Electrical and Computer Engineering, The Ohio State University, Columbus, OH 43210
}

Recent advances in two-dimensional (2D) materials and interfaces have opened exciting new possibilities for realizing highly tunable electronic properties through innovative band gap engineering at the interface. 2D materials can also be synthesized in a variety of compositions and structures, consequently resulting in novel properties at the interface that are not limited to the structural constraints in conventional interfaces, such as the choice of substrate and strain/defect control. Large area growth and/or transfer of 2D films have been extensively studied lately, most notably using metaldichalcogenides systems [1]. Here, we present the cross-sectional atomic scale imaging and analysis of GaSe 2D layered materials on GaN substrate using scanning transmission electron microscopy (STEM) [2]. Our analysis shows important details of the atomic structure that can dictate the electronic properties of GaSe and a new atomic scale reconstruction at the $\mathrm{GaSe} / \mathrm{GaN}$ van der Waals (vdW) interface that has not been observed in any conventional epitaxy. Our new findings may have important implications for controlling the atomic structure of $2 \mathrm{D} / 3 \mathrm{D}$ interfaces for tuning of the electronic structure at the interface for novel device applications.

GaSe can have multiple polytypes, and among them, the $\varepsilon$-type is a $\sim 2 \mathrm{eV}$ band gap semiconductor (Fig. 1a) and has been explored for applications in nonlinear optics, photovoltaics and photodetectors [3]. Our GaSe films were grown using a two-step temperature control in molecular beam epitaxy to ensure uniform film quality throughout the film structure. Cross-sectional TEM samples were prepared using focused ion beam, and examined using a $C_{s}$-corrected FEI Titan STEM operated at $300 \mathrm{kV}$. Despite the single crystallinity indicated by X-ray diffraction (Fig. 1b), $\beta$ domains are observed along with the intended, energetically more stable $\varepsilon$-type domains, forming stacking faults and domain boundaries in between (Fig. 1c). These two polytypes differentiate through a $60^{\circ}$ rotation of every other layer (Fig. 2). Rotational domain boundaries between the two types were also observed (indicated by the arrow). In addition, the $3^{\text {rd }}$ phase that had been previously unknown in literature was also found in between $\beta$ and $\varepsilon$ phases (Fig. 1c). The results indicate that precise control of phase and growth defects will be crucial to achieve the desired properties of these 2D films. The interface between GaSe and GaN showed the most unexpected structure. The truncation layer of $\mathrm{GaN}$ still retained the $\mathrm{GaN}$ surface reconstruction pattern ( 1 x 1 reconstruction), and the surface was also "decorated" with an array of dumbbell atoms at the top (Fig. 2a). While it is difficult to directly identify the composition of the dumbbell atoms, we believe those may be Se atoms passivating the GaN surface. This indicates that the structure of the vdW interface can be significantly different from conventional epitaxial interfaces. The new structure at the interface, such as the ones found in this work, could also be used to tune the electronic properties at the interface, for example, Fermi level pinning and dangling bond termination at the artificial 2D interfacial layer, potentially opening new exciting possibilities in the application of low-dimensional functional materials.

\section{References}

[1] S. Krishnamoorthy, et al., Appl. Phys. Lett. 109, 183505 (2016).

[2] C. H. Lee et al., (submitted) 
[3] Y. Zhou et al., ACS Nano, 8, 1485 (2014)

(a)

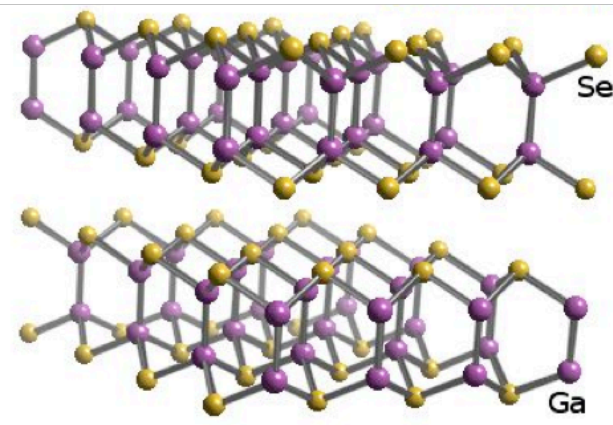

(b)



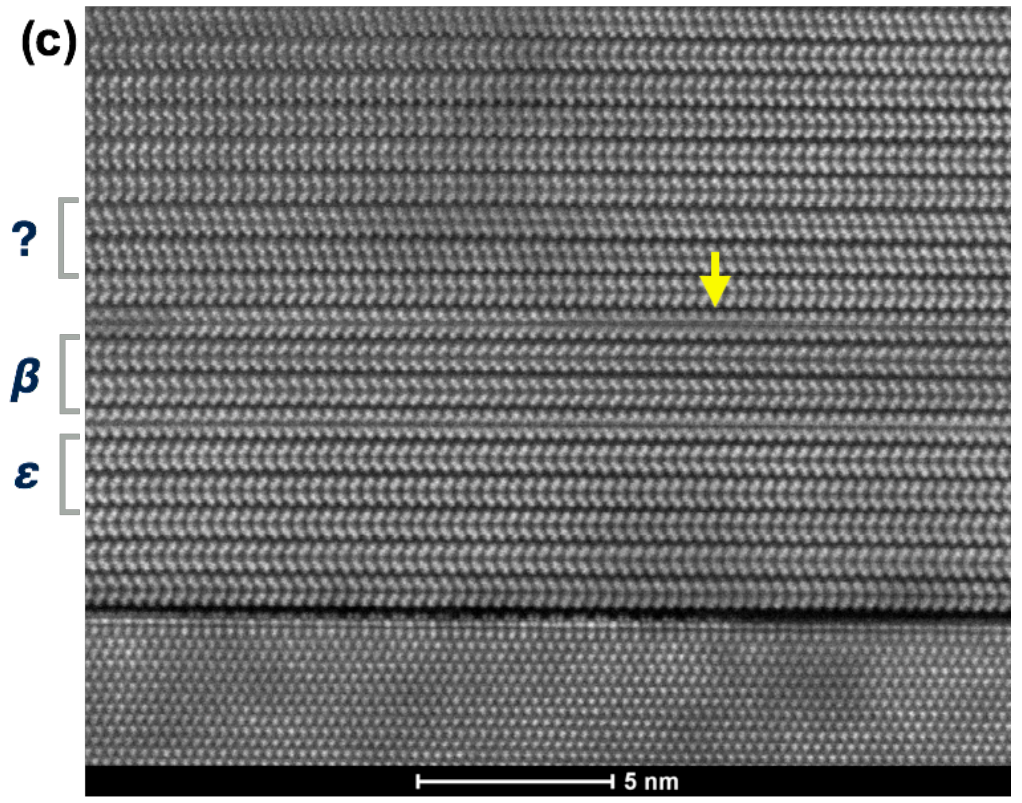

Figure 1. (a) Schematic of $\varepsilon$-GaSe. (b) XRD indicating good crystallinity of the GaSe on GaN. (c) Cross-sectional STEM image of the GaSe/GaN interface showing $\beta$, $\varepsilon$, and the unknown $3^{\text {rd }}$ phase. The arrow indicates the domain boundary.

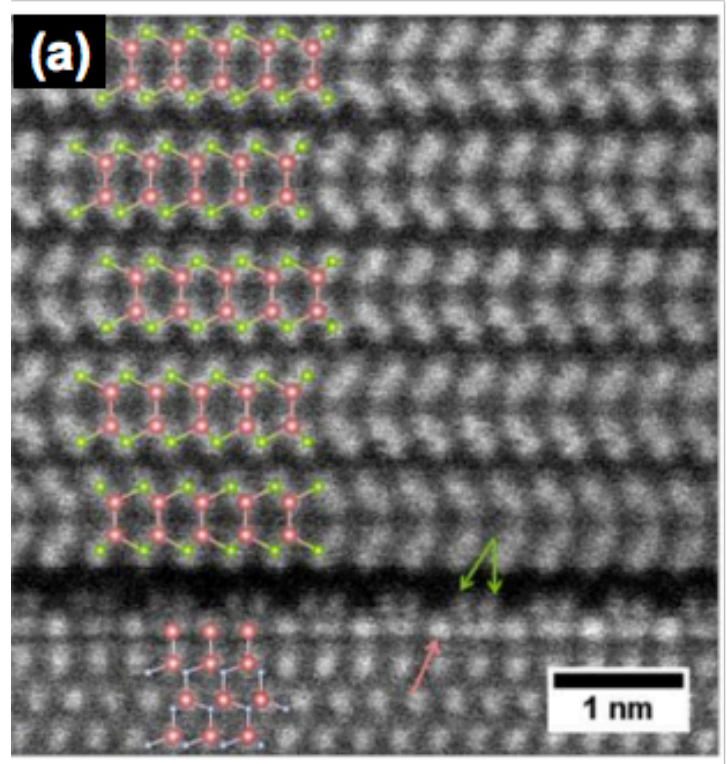

(b)

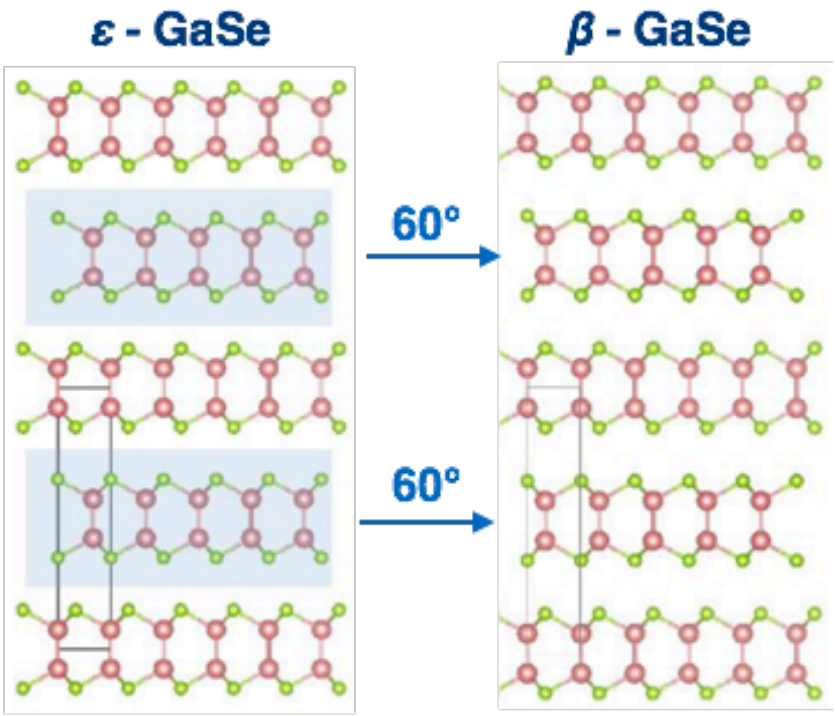

Figure 2. (a) Cross sectional HAADF-STEM image of GaSe / GaN interface. Indicated in the image are the GaSe layers including both $\beta$ and $\varepsilon$-type domains. Models of $\beta$ and $\varepsilon$ - GaSe are overlaid on the image. Reconstruction at the interface is also present, where the red arrow shows the $1 \mathrm{x} 1$ reconstruction of $\mathrm{Ga}$ and the green arrows show the dumbbell decoration. (c) Ball-and-stick models of $\beta$ and $\varepsilon$-GaSe types showing the difference in structure. $60^{\circ}$ rotation of every other layer in the $\varepsilon$-GaSe structure produces the $\beta$-type. 